Voix et Images

voixetimages

\title{
Pierre Perrault. L'envie de se taire
}

Yvan Dubuc et Yves Lacroix

Volume 3, numéro 3, avril 1978

Pierre Perrault

URI : https://id.erudit.org/iderudit/200116ar

DOI : https://doi.org/10.7202/200116ar

Aller au sommaire du numéro

Éditeur(s)

Les Presses de l'Université du Québec

ISSN

0318-9201 (imprimé)

1705-933X (numérique)

Découvrir la revue

Citer ce document

Dubuc, Y. \& Lacroix, Y. (1978). Pierre Perrault. L'envie de se taire. Voix et

Images, 3(3), 353-369. https://doi.org/10.7202/200116ar d'utilisation que vous pouvez consulter en ligne.

https://apropos.erudit.org/fr/usagers/politique-dutilisation/ 


\section{Pierre Perrault \\ L'envie de se taire*}

\section{ACCUEIL}

car j'ai l'histoire longue et la brette facile En désespoir de cause

Dans le domaine où j'exerce, bien que je vois un travail immense encore à faire, je sais pas comment... quoi faire exactement, je sais plus quoi faire...

j'ai l'impression de commencer à me redire, de plus savoir les chemins à prendre, d'avoir prolongé mon aventure trop longtemps dans le même sens...

au fond j'ai pas envie d'en parler...

pourquoi tu viens me parler littérature ? parce que c'est une revue litté-

raire, ton affaire...! je suis pas là-dedans...

puis je sais pas quoi dire!

je suis malheureux puis mal prêt...

Ce que je dis là,

Ce que je dis là, mis en exergue, c'est une espèce d'injustice par rapport à moi-même...

parce que, à partir de l'instant où j'ai décidé de me ramasser dans une affaire...

ou à partir du moment où j'ai une nouvelle inspiration... là, je viens d'avoir une idée de film... ça me travaille, à ce moment-là, on dirait qu'il y a plus rien qui pourrait m'arrêter...

il suffit que je parte!

* Entrevue avec Pierre Perrault réalisée les $1^{\text {er }}$ et 22 décembre 1977. La rencontre pour les commentaires a eu lieu le 24 janvier 1978. - Animation : Yvan Dubuc et Yves Lacroix. Transcription et montage : Yves Lacroix. - Nous présentons encadrés, afin de les distinguer de l'ensemble, les commentaires que Pierre Perrault a portés sur le montage final du texte de l'entrevue. [N.D.L.R.] 


\section{SUR LA FONCTION DE CINÉASTE}

le choix du métier, le doute, la folklorisation, le salon des métiers d'art, la possession du langage, la conscience, la tentation du silence

Mon aventure, elle est presque pas cinématographique, d'ailleurs je l'ai toujours dit que, pour moi, le cinéma avait pas une importance capitale...

il s'agissait simplement de trouver des moyens pour me permettre de faire une découverte, tu sais, c'est comme pour les chimistes, les outils, les instruments, la cornue, les pipettes, ç'a pas d'importance, ils vont faire des expériences avec ça ou avec d'autres choses...

puis peut-être que j'ai été emprisonné par le cinéma, parce qu'il s'est trouvé que le cinéma m'a permis de gagner ma vie et, un moment donné, ç'a cessé d'être un choix...

on a peut-être un certain nombre de disponibilités personnelles qui nous font prendre des instruments puis les transformer, faire quelque chose avec eux... de telle sorte qu'ils paraissent différents...

mais en définitive c'est la vie qui choisit pour nous...

je me sens un peu encarcané dans le cinéma... je suis pas certain que, si j'étais parfaitement libre, je ferais pas autre chose...

je suis à une espèce de tournant, d'hésitation... je suis obligé de réviser mes positions, de réfléchir sur ce que je vais faire demain... je suis pas sûr que ce soit à l'Office que je doive continuer...

mais je suis pas sûr si j'ai autre chose à faire...

ça fait trop longtemps que je fais du cinéma...

d'autant plus que le cinéma me paraît de plus en plus difficile...

parce que, d'une certaine façon, les premiers films que j'ai faits c'était les plus faciles à faire peut-être... c'était pittoresque, c'était gentil, c'était la couleur verbale, etc...

mais il y a pas rien que ça à dire... j'essaie de dire des choses infiniment plus difficiles à exprimer... mais qui ont besoin... qui méritent d'être exprimées, que j'ai le goût de pouvoir dire...

l'aventure qu'on a vécue il y a quelques années, ç'a été de vaincre le mépris, d'essayer de s'affirmer... de se découvrir une image, un homme... d'être, à travers les outils qu'on avait, cinématographiquement, quelque chose qu'on pouvait nommer québécois, bon!... ça, c'est fait! c'est pour ainsi dire accompli, cette aventure-là...

et peut-être qu'actuellement ce qu'on sait pas, c'est quelle est notre fonction dans l'élaboration d'une poétique, l'élaboration d'une culture ?

d'une certaine façon, je peux presque dire que je commence à avoir peur de la folklorisation...

la folkiorisation pour moi c'est l'occupation du territoire de la mémoire quand tous les autres territoires sont abandonnés...

et j'ai le sentiment d'être devenu, à cause du contexte de la société, qui est absente de son propre territoire, qui est subventionnée, qui est 
engagée, qui est employée... par d'autres... d'être une sorte de sculpteur de Saint-Jean-Port-Joli... qui fabrique des beaux objets... pittoresques, sympathiques, chaleureux... des objets de causerie, des objets de parolis, des belles parlures, toutes choses qui sont de vieux vêtements, qui sont des combles pointus à coyaux

qui sont des tendresses qu'on peut avoir à notre propre égard...

mais pendant ce temps-là, les choses importantes de la culture, c'est-àdire le pain puis le beurre... personne de notre parlure ne s'en occupe, donc on n'a plus de territoire à occuper...

on est en train de folkloriser le Québec, on lui fabrique des chansons puis des poèmes... mais le Québec n'a aucune maîtrise... le Québec est réduit à sa boîte à lunch...

puis il écoute des gens qui lui racontent des histoires merveilleuses d'un peuple... de gens ẽxtraordinaires, des Grand-Louis, des Alexis... il se dit Je suis bon, je suis fin

mais le seul jour de l'année où il est québécois... justement aujourd'hui je suis allé au salon des métiers d'art... là il s'en va s'acheter une petite tasse québécoise puis un petit gréement québécois, il paie un prix fou, c'a pas de maudit bon sens !...

mais il mange toute l'année dans la cuisine japonaise, dans de la vaisselle japonaise... il achète du Swift puis du Prem puis du Klick puis du Klam... puis du baloney...

il occupe pas le territoire de la culture, le pain, là, la fabrication du pain, il a abandonné le goût du pain à son meilleur ennemi...

c'est cette espèce de vacuum incroyable qui fait que les gens hésitent devant leur propre destin... ils se regardent les mains puis ils disent Qu'est-ce que je vais faire avec mes mains? je' sais pas faire ça!

je me reprends à resonger à ma préface de l'Acadie du discours... notre lieu c'est la parole, notre pays c'est le langage... et aussitôt qu'on quitte le langage... on est chez les autres... et c'est ce qui me rend, jusqu'à un certain point peut-être actuellement, la parole pénible... effarante...

il y a eu des périodes de ma vie où je me suis senti physiquement... comme vidé, je pense d'ailleurs que le printemps dernier, quand j'ai écrit Trou d'homme, j'ai ressenti physiquement l'angoisse qui était très bien exprimée dans le message d'Hubert Aquin, je la sentais moi aussi... et ça, je pense que c'est physique...

actuellement je suis pas du tout dans un état semblable... je me sens très vivant... j'ai le goût de faire des choses, j'ai le goût de vivre, de rencontrer des gens, de parler, d'aller au théâtre même... la chasse... d'être heureux, pour mon propre compte... mais c'est ma fonction de...

qu'est-ce que c'est, ma fonction ? je sais pas... ma fonction poétique...

j'ai quand même écrit de la poésie, ç'a peut-être pas beaucoup de valeur mais tu te valorises du simple fait que tu essaies d'en écrire, tu te donnes un rôle à ce moment-là, bon ! 
ce rôle-là, j'ai de la difficulté à le tenir parce que je le trouve presque anachronique dans la société, je trouve qu'on essaie de retenir quelque chose par la peau des dents... tu sais, qu'on est en train de tomber puis qu'on est accroché par les doigts puis les doigts commencent à être fatigués puis...

de fatigue on est en train d'abandonner ça...

c'est extrêmement curieux que je dise ça à une époque où on pourrait croire que la parole est au pouvoir... une parole que j'ai soutenue longtemps... mais je pense qu'une part de mon pessimisme vient du fait que je sens que les gens attendent des réponses politiques...

j'ai une espèce d'angoisse...

les hommes qui ont voté pour une libération... ils l'attendent... ils ne la vivent pas..

regarde ce qui se passe actuellement... ce qui se passe actuellement c'est pas une chose banale... tu comprends, il y a quand même un changement de gouvernement qui dit qu'on va se prendre en main par tous les moyens...

puis tout à coup t'as je-sais-plus-quelle-centrale-syndicale qui est furieuse parce qu'on a pas forcé les multinationales à rouvrir la Wayagamack...

baptême ! la Wayagamack, il faut surtout pas qu'il y ait de multinationale là-dedans ! il faut la prendre, tu comprends ! il faut le faire, notre bois ! faut le faire, notre papier !

parce que, tant que les autres le font à notre place, tout ce qu'on a, nous autres, c'est la boîte à lunch... puis les gens demandent des boîtes à lunch!

ce que je dis, moi, aux gars de la Wayagamack Prenez-le, le pouvoir! prenez-les, les arbres!

il y a un pays à bâtir, bon yeu ! puis le monde veut garder ses petites planques, sa petite pitance...

puis l'imagination travaille pas, on dirait qu'il y a personne qui veut entreprendre le pays... ils l'attendent... ils veulent la sécurité...

j'ai l'impression de parler pour rien dire... j'ai l'impression d'être, non seulement au niveau de ma parole quotidienne avec les amis là... mais aussi au niveau de mon travail de cinéaste ou d'écrivain... d'être une conscience qu'on veut pas avoir, qu'on refuse d'avoir...

c'est pas être conscient de ce qui se passe, c'est pas essayer de se libérer dans son âme, de se fabriquer, de se charpenter une âme libre que les gens veulent, c'est d'être libérés par d'autres ! autrement dit, on attend toujours les trois navires

et les Québécois comme Alexis ils seraient prêts à ce qu'on les délivre mais ils veulent pas se libérer eux-mêmes...

un moment donné, cette parole-là que tu as entretenue... tu lui as donné les assises de ton goût des hommes... de ton orgueil... de ta connais- 
sance des hommes que t'as rencontrés autour de toi... cette parole-là devient insupportable...

moi je me sens une terrible envie de me taire...

j'ai pas le moyen !... est-ce qu'on peut se taire quand on vit de sa parole ?... puis je peux pas me taire non plus, pour une raison ridicule... c'est que je suis tellement habitué à travailler, ça fait si longtemps que je travaille pour faire mes films, enregistrer mes bandes sonores, transcrire, que, même quand je suis fatigué de travailler... je m'ennuie, parce que je travaille pas... je ne saurais pas... je sais pas comment me reposer... comment garder le silence... je sais pas comment faire ça...

C'est pas dans la mesure où je veux parler, mais dans la mesure où je veux entendre la parole des autres... et cette parole est plus mienne que la mienne...

autrement dit, j'ai l'impression de mieux me reconnaître, de mieux me retrouver dans ce que je fais dire aux autres, dans ce que je permets aux autres de dire, que dans ce que je dis moi-même...

pour me vérifier, pour trouver mon propos, j'ai toujours besoin d'entendre les autres...

écouter, tu sais...

\section{SUR GÉLIVURES}

la non-précarité, la victoire, l'être collectif, la force, le seuil de grégarité, la chefferie, les communications

Ce livre-là cherche à raconter la formation des œkoumènes, c'est-à-dire qu'on part du rien, on part de la roche nue après le passage du glacier et, progressivement, arrive, intervient le lichen colonisateur qui gruge un peu la pierre, qui fabrique un humus avec les siècles d'érosion, de vent...

puis sur cet humus mince s'installent des lichens un peu plus riches comme les cladonies, puis tout à coup apparaissent les caribous qui commencent à brouter puis le chasseur derrière...

et puis la société derrière, et puis une société qui s'organise un peu plus, l'intervention des clans bien sûr...

et puis tout à coup l'idée d'un royaume qui s'installe, c'est-à-dire le rapport d'une collectivité avec un territoire par lequel ils se nomment...

c'est le mot qu'on a inventé, il y a quelque temps, le mot Québec...

je l'ai écrit dans le livre de René Lévesque...

Option Québec...

j'ai.écrit le dernier texte... le premier mot que j'ai dit c'est le mot Québec c'est un mot qui grandit...

pour beaucoup de gens, le fait de le lire, ils se sont aperçus...

puis moi, le fait de l'écrire, je m'en suis aperçu aussi, à ce moment-là

il existait, il était dans l'air, quelque chose changeait progressivement, on était entraîné par ça... 
cette tentative d'acheminer l'occupation du territoire à une perfection

qui nous donnerait ce qu'on appelle la souveraineté puis ce qui est

le passage d'un état précaire à un état plus stable... à un État justement...

C'est ça la lutte, le combat incessant de l'homme vers la non-précarité... toutes les images qui sont là-dedans, autant l'image de l'iglou, l'image de mes oncles cultivateurs, l'image de la misère en Abitibi, tendent à acheminer l'homme à un moment... le seul cheminement... le chas de l'aiguille par lequel on pénètre dans la stabilité, la sécurité... c'est la victoire ! la victoire du bœuf musqué...

et je ne vois pas d'autres cheminements... il y a pas d'autres moyens d'arriver à cette plénitude de l'être collectif, le rapport avec un territoire, par lequel tu te nommes... souveraineté... et ça tu y parviens que si tu es le plus fort...

et je ne vois pas ailleurs, nulle part ailleurs d'autres moyens... j'implore! je dis Qui inventera d'autres tables de la loi ?... mais toute ma réflexion, à partir des clans jusqu'à l'homme d'aujourd'hui, m'oblige à arriver à cette conclusion-là... qu'il n'y a que la victoire... il n'y a que la force qui te donne droit à toutes les vaches du troupeau, et à ce goût d'herbe qui est incomparable...

les confédérations, les constitutions, les traités de paix, toutes les lois des hommes sont des accommodements avec une loi que les hommes n'ont pas énoncée... qui est une loi quasi biologique...

les cheminements vers cette plénitude-là passent toujours par le même chemin du combat...

on pourrait imaginer 50 formes plus simples, mais il faut qu'il y en ait un qui meure, il faut qu'il y en ait un qui dénonce sa force...

la violence, c'est quelque chose qui me déplaît... même si je suis prêt à regarder le combat des bœufs musqués

mais si je domestique le bceuf musqué... je vais éviter qu'ils se combattent... je vais en mettre rien qu'un parmi les femelles...

mais à partir du moment où il n'y a personne qui nous domestique, qui réussit à nous dominer, c'est fatalement ce qui nous attend... ou presque fatalement... le combat pour la possession de la harde...

il faut que tu atteignes le seuil de grégarité, c'est une de mes grandes préoccupations, est-ce que les Québécois ont atteint le seuil de grégarité ? est-ce qu'ils sont assez nombreux pour former un troupeau autonome?

quand deux troupeaux de caribous se rencontrent, le plus petit se met à suivre le plus gros... il a des phénomènes d'entraînement, une sorte de fatalité dans l'ordre de grandeur...

le seuil de grégarité, c'est une question de nombre par rapport à la masse voisine... tu comprends, tu pouvais avoir les Iroquois, quand il n'y avait pas les Américains, les Iroquois existaient... 
puis comme la masse qui était à côté d'eux, les Algonquins, étaient en équivalence, ils pouvaient voisiner, coexister..

quand les Blancs sont arrivés, qu'est-ce qui est arrivé de toute la civilisation amérindienne?

pourquoi?

on fait un procès, on dit : Les Blancs, le commerce des fourrures c'est vrai tout ça... mais ça, c'est des détails... je l'ai dit dans Gélivures un moment donné... ceux qui ont aboli la torture sont également génocides... parce que la structure de leur société, les Amérindiens, leur force... ils savaient que la force était importante... elle était rituellement construite sur ces phénomènes-là...

tu les a privés de leur façon de se mettre en rapport avec l'univers... ils se sont complètement anéantis à ce moment-là...

maintenant, tout à coup, ils retrouvent leur façon, puis ils se mettent en lutte avec nous autres...

C'est assez obscur...

ça se traduit pas toujours par des mots, la parole!

la société amérindienne était construite sur une morale dont:le cérémonial était la torture...

ils savaient que leur force était fondamentale, ils en avaient besoin pour survivre... à partir du moment où on a aboli la torture, ils ont perdu leur force, leur capacité de résister...

il faut une chefferie!

puis une chefferie c'est pas rien qu'un chef de bande... une chefferie c'est un chef de bande là où il y a des bandes...

une chefferie c'est pas un chef de bande quand il y a des sociétés...

Quand il y a une société, le chef de bande n'est pas un vrai chef... il faut un chef de société...

c'est pourquoi la société québécoise a raté son évolution...

c'est que, par des trucs politiques, on l'a plafonnée au foreman... puis au secteur service ! des docteurs, des médecins puis des avocats...

mais c'est pas une société ça... la société pouvait pas avoir la conscience de sa totalité, puisqu'elle ne possédait pas les rouages administratifs, intellectuels... et de communication, le pire c'est qu'on a perdu la communication...

tous ces rapports-là nous ont échappé, c'est d'autres qui s'en occupaient... 
On avait des chefs de bande, des foremans, des curés... il n'y avait pas de vrais chefs de la société...

le gouvernement qu'on avait. c'était un gouvernement partiel, il ne gérait qu'une partie de cette société-là, d'une part... d'autre part, il était facilement complice d'un autre système... parce que c'était un gouvernement de notables qui cherchaient à préserver leur notariété, leur confort...

ils avaient appris que la société, c'était autre chose que cet ensemble d'individus qu'on disait avec beaucoup de mépris les habitants...

ils ont pris leurs modèles ailleurs !

il faut que le peuple progressivement s'empare de son territoire...

tu sais même pas que les choses existent... tu sais même pas ! ton fils, il sait pas, toi, qu'il peut devenir géologue, qu'il peut devenir ingénieur en métallurgie, il le sait pas parce qu'il sait même pas qu'il existe quelque part des endroits où il y a des ingénieurs qui travaillent sur les métaux, il le sait pas... comment est-ce que tu veux qu'il le devienne?

4. SUR LA DOMESTICATION le pays, l'urgence, le pouvoir d'achat, l'occupation du territoire, les dauphins bleus et la retraite en Floride, la conscience collective, l'exemple dẹ l'architecte, l'état de siège

J̀e suis comme prisonnier d'une démarche qui était amorcée en moi puis dans la collectivité...

je suis prisonnier d'un propos en quelque sorte... propos qui est difficile à soutenir parce qu'il est très passionné...

tụ peux très bien décrire... l'épinette rouge dans le vent, l'automne, quand elle est jaune... calmement, tranquillement, mais j'ai pas envie de ça ! j'ai pas envie de cette forme poétique! même de mon rapport personnel avec le paysage...

je n'ai envie et je ne peux parler que du pays... je suis prisonnier de cette affaire-là...

il y a une phrase, qui n'est pas extraordinaire mais que j'aime beaucoup parce qu'elle décrit ma situation en quelque sorte... de Jacques Ferron dans Escarmouches...

Dans un pays comme le nôtre, qui n'a pas été dit ça, c'est peut-être pas tout à fait vrai... il faudrait dire: pas assez dit pour être accompli... le sujet de l'écrivain c'est le pays lui-même...

et ce qu'il y a de terrible c'est que ceux qui parlent pas de ça, ils m'ennuient...

parce qu'il y a une telle urgence, que je veux pas entendre parler d'autre chose... quand j'ai faim, viens pas me parler de musique... c'est obsessionnel, c'est suicidaire aussi, parce que ça devient une hantise...

on est assiégé de toutes parts, c'est cette urgence-là que j'essaie de faire partager au monde puis que personne veut comprendre... 
c'est dur à porter seul puis ça rend... ça rend... agressif peut-être, je sais pas quoi, je sais pas comment dire ça...

il y a une espèce de mécanisme, un rouage de la pensée qui fait que, à partir de tout ce que je vois, de tout ce que je rencontre... je nourris une volonté de changer le monde, de changer ce monde-là, puis j'essaie de l'exprimer cette volonté-là, de toutes les façons possibles...

puis c'est ça qu'on veut pas... on veut pas m'entendre crier...

mes nièces, mes neveux, ils disent $A h$ ! encore mon oncle qui chiale! tu sais qui est pas content!

quand je vois 70000 personnes regarder la coupe Grey... ils aiment ça! ... je leur dis Ces gars-la sont juste venus plumer l'alouette !... l'alouette qui est là c'est vous autres ! vous êtes en train de vous faire plumer !... vous êtes en train d'enrichir je sais plus qui, je sais pas quoi... mais vous êtes en train de vous faire endormir!

on aurait d'autres choses à faire de plus important... on aurait à prendre possession... à inventer... on est là pour recommencer le pays au premier arbre...

puis les gens sont pas conscients de ça, ils attendent la réponse du gouvernement puis, en attendant, ils vont au football...

on a un seul maudit pouvoir puis on l'exerce pas ! c'est notre pouvoir d'achat !... c'est simple comme bonjour, on peut permettre des choses avec ça, mais faut bậtir...

tu sais, nous-autres, les poètes, les fous, on a bâti l'idée d'un royaume... maintenant il faut rebâtir cette idée-là dans la soupe... dans les confitures aux fraises!

je dis ces phrases-là devant du monde, à Jonquière ou à Sherbrooke...

ou au collège Marguerite-Bourgeois... il y a des femmes qui se lèvent dans l'assemblée puis elles viennent m'apporter un pot de confiture aux fraises qu'elles ont fait dans leur maison... c'est notre dernier refuge !...

je leur dis Vous faites rien ? ils viennent m'apporter leur confiture aux fraises, ou leur confiture aux prunes... il leur viendrait pas à l'idée de mettre leur nom là-dessus puis le mettre sur des tablettes...

toi-même tu t'habilles comme un Américain ! on est tous de même !... on est costumé intellectuellement, physiquement, par des cultures en dehors de nous autres, parce qu'on n'a plus rien pour s'inventer, on n'est plus capable de s'inventer un costume, un chandail, une chemise, une tuque même... c'est pas des farces, c'est hallucinant quand tu vois ça...

les peuples autrefois faisaient leurs chaises, leurs tables, leurs maisons, tout ça, il y avait toute une structure mentale, complète, totale, par laquelle ils pouvaient s'approprier leur être... c'est insignifiant mais qu'est-ce que tu veux dire sauf quelque chose de dérisoire?

quand t'es rendu que tu bâtis en plein fleuve Saint-Laurent un aquarium et que tu mets dedans des dauphins bleus de Floride, alors qu'il y a des marsouins dans ton fleuve... t'es capoté complètement ! 
monsieur Alcan qui l'a subventionné, qui a donné des sous, lui, il est américain, c'est dans son pays aussi, les dauphins bleus de Floride, mais pas nous autres.

on est en état d'urgence, on est en train de perdre la guerre économique... faut pas s'imaginer qu'on n'est pas en guerre... ils sont après nous racheter...

ils ont racheté tous nos boulangers puis ils les envoient finir leurs jours en Floride, y a-t-il quelque chose de plus triste que ça ?...

manger des pamplemousses !...

je suis certain quie les gars apportent une chanson de Gilles Vigneault en Floride, puis ils écoutent ça sur la plage, puis Gilles Vigneault leur parle de Natashquan, puis il. leur dit Mon pays c'est l'hiver puis ils trouvent ça beau !

c'est ça qui m'hallucine moi!... ça prend, pour résister à ça, un effort de conscience extraordinaire!

je te dis pas qu'on est pas en train de le faire, je te dis pas que c'est pas exemplaire et héroïque et extraordinaire, ce qu'on fait... mais je suis sûr que ça va réussir...

c'est dans le quotidien qu'il faut faire quelque chose... c'est ça que les gens n'arrivent pas à comprendre, puis quand je leur dis ça je les dérange...

je m'en vais te raconter une histoire.. c'est la plus belle que je connaisse...

c'est mon beau-frère... je l'aime bien, c'est mon meilleur ami... il marie sa fille... bon ! je vais y aller c'est bien sûr...

il m'invite un soir, il dit. On va gooter le vin de la noce... il y avait trois ou quatre sortes de vins mousseux d'ici puis de là, ils étaient bons ! ils étaient convenables !...

j'ai dit Pourquoi tu prendrais pas du cidre? d'abord tu prends pas de champagne, c'est trop cher, t'as raison ! c'est pas meilleur ! un mousseux, c'est bon ! puis du cidre, du Fernand-Dufour, c'est aussi bon, puis c'est québécois, deux bonnes raisons... ils ont ri de moi un peu...

il est architecte, lui... tout à coup j'y pense... j'ai dit René, sais-tu... je comprends asteur pourquoi ils ont pris Taillibert...

le maire Drapeau, quand il achète, il achète du champagne ! il fait venir Taillibert! il achète pas québécois !

encore une fois, c'est ce que je répète, c'est ç̧a mon affirmation... on est en état d'urgence, en état de siège, quand t'es en état de siège, t'ouvres pas la porte à l'ennemi... tu gardes tes armes... tu gardes ton blé, tes réserves, tes provisions...

si un peuple possède pas une certaine quantité de maîtrise, il est aculturé, parce que la culture commence avec le pain... la chanson c'est à l'autre bout que ça arrive...

nous autres on fait actuellement des chansons parce que nos pères avaient une culture, mais nos enfants font des chansons sur la Californie eux autres... les merveilleux enfants qui ont fait Parti pris, ils nous proposent le Prince de Sexamour puis ils mettent des robes blanches comme des Hindous... 
c'est là que tu vois que t'es occupé, il y a un impérialisme de toute part qui t'occupe parce que t'as créé le vide en toi...

puis cette espèce de vide-là... je commence à penser que la parole ne peut plus le remplir...

\section{SUR}

\section{L'ABITIBI}

le projet de film sur la baie James, la route La Sarre-baie James, le royaume qui attend, Hauris Lalancette, l'échec, le cochon qu'on égorge

Je suis allé en Abitibi...

en proposant un film sur la baie James...

j'avais une idée de film...

je suis parti d'une idée abstraite, de l'idée qu'il y avait eu la Manicouagan et que ç'avait été une grande affaire...

La Manicouagan, ç'avait été une grande affaire... c'est une hypothèse de royaume que le Québec avait entreprise, avait conçue...

et puis ça s'est passé... puis il y a eu des émotions, il y a eu des chansons...

il y a eu un résultat ! le Québec pour une fois avait un monument qu'il avait construit de ses mains... de ses ouvriers, de ses foremans mais aussi de ses ingénieurs... il avait vraiment conceptualisé toute l'affaire...

je suis parti avec cette idée-là... et je suis arrivé à Matagami...

j'ai rencontré des électriciens, des contremaîtres, des ingénieurs de

l'Hydro-Québec, des ingénieurs des compagnies qui étaient là...

puis j'ai cherché quelqu'un qui était là.:.

puis j'ai cherché quelqu'un qui était là avec l'idée de royaume...

c'est toujours ça; la conquête, que je cherche...

je sais qu'on n'a rien! alors la moindre parcelle de terre qu'on réussit à conquérir, la moindre petite maîtrise, le moindre territoire, c'est toujours ça de pris... c'est ça que je cherchais...

les gars, ils prenaient du scotch puis ils parlaient des femmes... ils avaient hâte de revenir à Montréal, j'ai pas trouvé la moindre étincelle en faveur du royaume... je dis pas qu'elle n'existe pas ! je dis Je ne l'ai pas trouvée...

je me suis emmerdé, j'avais pas envie... je fais jamais de film avec des gens que j'aime pas, des gens avec qui je peux pas communiquer, j'ai pas d'émotion, de passion...

puis je suis parti avec un bonhomme là... le gars avait quand même un petit sentiment de quelque chose, il m'a amené à La Sarre...

puis là j'ai rencontré Dominique Godbout, un notaire... responsable d'une idée complètement folle et de son exécution... la route La Sarre-baie James...

complètement loufoques, les gars ont décidé... ils partaient l'hiver pendant le temps du chômage... dix-sept hommes puis des bulldozers, puis ils se sont rendus jusqu'à Fort-Rupert... complètement craqués, c'est 
fantastique ! parce que c'est épique et inutile... ç'a jamais servi à rien mais ils espéraient que ça serve à quelque chose...

puis là, il m'a présenté Charlemagne Gobeil, celui qui déménageait les maisons... là je me suis trouvé dans quelque chose ! l'Abitibi qui déménageait puis le gars qui faisait une route pour la conquête du Nord ! lui il avait envie du Nord !...

il se trompait ! mais il y avait du désir en lui !...

j'ai été voir ce monde-là, ce monde-là était vivant... imparfait ! incapable de faire des projections sur l'avenir ! aucun pouvoir !... ils connaissaient pas les possibilités, puisque, je te dis encore, c'est du monde plafonné au foreman, ils savaient pas ce qui s'en venait...

mais ils avaient quand même une passion pour le royaume... la suite de l'Abitibi, puis Si ça marche pas on va aller plus loin ! on va trouver! s'il faut cultiver les arpents de neige, on va les cultiver!

c'est pour ça que je suis allé vers l'Abitibi...

dans l'Abitibi j'ai trouvé le gars le plus extraordinaire... le cultivateur le plus fantastique que tu puisses imaginer...

les cultivateurs, eux autres, ils cultivent leur terre... lui, il cultive pas la terre, il cultive le royaume... pour lui, un morceau de terre c'était pas un morceau de terre, c'était un morceau du Québec... puis il fallait gagner ce morceau de terre-là pour faire le royaume parce que les curés puis Duplessis leur avaient dit Un royaume vous attend... lui it est encore là pour le royaume...

le sentiment de la conquête, l'incarnation même du goût du royaume que j'ai toujours poursuivi dans tous mes films, c'est dans Hauris Lalancette que je le trouve, avec une naïveté, si tu veux, mais une splendeur en même temps... c'est vraiment épique... tu comprends, c'est Éric le Rouge... Hauris, avec toute sa verdeur puis sa truculence, sa philosophie... c'est absolument fantastique ce personnage-là !

il est le résultat historique d'une série de phénomènes... élevé au marché au foin à Saint-Hyacinthe, embarqué dans le Crédit social...

il ramasse toutes les carences, les défauts, les difficultés, l'ignorance... puis en même temps un génie du verbe... une verdeur, une volonté, il y a des millions d'affaires dans ce gars-là, c'est un méli-mélo québécois incroyable... tout est là...

et sa conquête me paraissait importante à dire parce que c'est celle de la terre...

c'est pas parce que je privilégie la terre - il faut se nourrir, c'est une des fonctions - mais parce que la terre est un symbole, comme le pain est un symbole...

si les gens pensent que je préconise un retour à la terre comme le voyait Alexis, ils se trompent grossièrement, mais c'est peut-être parce que mes films sont mal faits...

vois-tu, j'ai fait en Abitibi l'histoire de la mort de l'Abitibi...

à travers un gars qui a refusé de mourir, qui refuse encore de mourir... il va mourir pareil! 
il est pas mort, mais il va mourir, il peut être tout seul, ç'a pas de bon sens... pour se justifier à la face du monde, il faudrait qu'il fasse une clôture tout le tour de l'Abitibi puis qu'il emplisse ça d'animaux...

il peut pas, dans son champ à lui, dans ses cinq ou six cents acres, continuer à vivre... ils vont lui couper la route, ils vont cesser d'entretenir les chemins...

I'histoire de l'Abitibi, c'est la dernière grande aventure collective avant celle qu'on entreprend maintenant... les Québécois...

c'est un échec incroyable...

puis c'est une leçon pour ce qu'on essaie d'entreprendre, parce qu'on peut se jouer le même tour... exactement le même tour...

et le tour que l'Abitibi s'est joué, c'est que toutes les superstructures n'appartenaient pas à l'Abitibi, toutes les structures commerciales, technologiques, etc... les marchands sont allés exploiter l'Abitibi, ils l'ont vidée de sa substance... et les boîtes a lunch sont restées des boîtes à lunch!

Les cultivateurs qui avaient réussi à ouvrir les terres, qui avaient réussi pendant un certain temps à vivre assez bien sur ces terres-là... à cause du commerce se sont sentis appauvris, se sont sentis incapables de vivre dans le système qui leur était proposé et ils sont retournés à la boîte à lunch!

Hauris, il crie, il hurle...

il épargne personne...

la mort c'est pas drôle... quand tu tues un cochon, il chiâle ! c'est un cochon qu'on égorge...

le monde est pas habitué... d'habitude le cochon qu'on égorge, il est incarné par Alain Delon...

mais moi c'est des films que je suis content d'avoir faits, je regrette pas de les avoir faits...

des films grinçants... des films que les gens aiment pas, parce qu'Hauris, il crie, il hurle... c'est un cochon qu'on égorge...

\section{ENCORE}

\section{SUR GÉLIVURES}

l'inutilité, le silence, la futilité, l'indépendance pourtant, le silence, l'inefficacité, l'ostracisme, la tentation du silence

Moi j'ai écrit ce livre-la... j'étais content de le publier actuellement, dans le contexte politique qu'on vit parce que c'est un livre sur la conquête du royaume...

je me dis Bon ! voici une substance à la réflexion du Québec actuellement ! je m'attends pas à ce que madame Chose n'importe où le lise... mais je pensais mettre ma pierre dans le mur...

mais ils l'ont laissée là, à côté, la pierre... je suis un petit peu déçu de ça, mais je suis pas déçu dans le sens que j'y tenais, je suis déçu dans le sens que je pensais que ça pourrait servir...

j'ai l'impression d'avoir perdu mon temps... 
on dirait que le propos échappe maintenant... que le propos doit s'en tenir à des législations, que la poésie est là... dans la politique...

peut-être que la nationalisation ou l'achat d'une compagnie d'amiante, c'est l'acte poétique par excellence aujourd'hui, et que tout le reste c'est tellement futile !... à partir du moment où t'as le pouvoir en quelque sorte...

pourtant il reste un domaine que je-trouve inoccupé par la conscience collective actuellement, c'est le domaine même de la conscience collective...

et du besoin de mettre l'épaule à la roue... chaque individu... moi j'ai pour mon dire... le Parti Québécois fera l'indépendance quand l'indépendance sera un fruit mûr puis il pourra mettre la main en dessous...

or qu'est-ce qui va faire mûrir l'indépendance ? c'est l'indépendance en chaque individu... et ce travail-là je vois pas qu'il soit fait par personne, dans les individus...

moi j'essaie de le faire...

après avoir bâti l'orgueil, je suis presque au point où je vais essayer de bâtir la volonté de faire...

on dirait que c'est plus possible...

je me sens repoussé... repoussé à la fois dans mes préoccupations, dans ma volonté de gueuler pour rendre compte de cette urgence-là...

pourquoi personne a parlé de Gélivures ?... pourquoi personne a parlé de mes films dans les journaux?

j'ai eu juste une réponse... j'ai été invité par Alain Bienvenue, professeur au collège Jean-de-Brébeuf, à aller rencontrer ses étudiants...

il a mis Gélivures au programme...

je te dis que je me suis pas fait aimer par les étudiants !

les gars étaient découragés !

ils trouvent ça difficile!

c'est une convergence de silence actuellement... qui rend un son de cloche que j'arrive pas à analyser...

je me sens sans allié parmi mes ennemis, puis sans défenseur parmi mes amis, dans une situation où je suis quasiment interdit...

même si on m'autorise à faire des films, mes films ne sortent pas ! sauf dans des festivals en France !

ça m'intéresse pas de savoir ce que le reste du monde pense de ce que je fais...

c'est ici que je parle au monde...

j'avais le contact avec le public ! là je l'ai perdu !

je sais pas pourquoi mais je l'ai perdu...

je ne veux pas accuser personne, j'aime mieux pas accuser personne, mais c'est des faits historiques ? des circonstances ? c'est quoi ? c'est que mes films sont moins accrocheurs qu'autrefois? je sais pas quoi... 
peut-êre parce que c'est une vieille tonalité puis la chanson a été assez chantée...

j'accepte d'en parler quand quelqu'un m'en parle, mais depuis au-delà d'un an que Gélivures est publié, une fois seulement j'en ai parlé autant que ça... en France, j'ai eu deux heures de télévision pour en parler... puis maintenant, quelqu'un m'en parle pendant un moment...

moi maintenant, il existe plus, le livre, j'y pense plus, je fais autre chose, j'arrête pas de travailler pour autant, je fais un autre livre, d'une autre forme, sur le même sujet d'une certaine façon...

mais je me dis... dans le contexte qu'on vit, si j'avais écrit ça dans une forme plus efficace au niveau de la communication, peut-être que ça aurait été efficace aujourd'hui...

je le sais pas... je le sais pas...

je suis pas sûr...

i) faut quand même le rendre accessible, c'est ça la communication hein ! une route c'est quoi ? c'est pour rendre accessible... un poème c'est pour rendre accessible aussi...

je te dis... les étudiants du Brébeuf l'autre jour, 16-17 ans... ils auraient voulu m'étriper, ils disent On comprend rien !...

ils comprennent les chansons de Gilles Vigneault, donc Gilles Vigneault communique mieux, il rend plus accessible...

Gélivures c'est le livre que je suis le plus heureux d'avoir fait..:

c'est là-dedans que j'ai mis toutes mes complaisances...

ce que j'ai raté, ce que je regrette d'avoir raté c'est la communication...

puis je suis malheureux parce que...

tu t'asseois à la table pour écrire ou tu parles avec des gens, c'est la même chose au fond...

je parle, c'est pour que tu me comprennes...

puis moi je m'emmerde avec les philosophes parce que c'est pas mon langage...

qui parlent un langage que je comprends pas, que je peux pas suivre... ils m'écœurent, ces gars-là...

alors que moi je parle un langage que je comprends mais j'écœure du monde puis ça me fout en boule!

je suis malheureux de ça!

les petits gars qui étaient devant moi l'autre jour, j'avais envie de leur parler... j'ai pas été capable...

j'ai été incapable de leur dire ce qu'il y avait dans ce livre-là...

parce que je sentais que la barrière était tellement grande que je pouvais plus communiquer avec eux...

remarque, je leur ai dit une chose... j'ai dit Moi quand j'avais 16 ans j'ai lu l'Abatis puis j'avais exactement la même réaction que vous...

je l'ai relu plus tard puis ça reste pour moi un des grands livres, sinon le plus grand livre québécois... je dis québécois dans le sens que c'est une écriture qui a un rapport avec le Québec... 
alors ils ont 16 ans... mais j'ai l'impression...

ce que je sais, c'est que tous ceux qui portent, ou qui partagent une certaine idée de royaume, sont ostracisés... il y a un complot de silence autour d'eux...

regarde jusqu'à quel point le Québec est occulté dans le Devoir actuellement...

comme jamais ! ça n'a jamais existé, on pourrait faire un inventaire...

les sociologues devraient prendre le Devoir de cette année puis le Devoir d'il y a deux ans puis faire des inventaires pour voir la différence...

c'est volontaire ou c'est inconscient mais c'est tragique... c'est tragique...

de sorte que ce qui existe actuellement au Québec, son existence, ses affirmations, ne parviennent pas à la conscience des gens...

et ma peine à propos de ça, c'est pas parce que moi personnellement je suis engagé là-dedans, mais je m'aperçois que c'est comme ça sur tous les terrains... c'est comme ça partout...

J'ai l'impression que l'idée de royaume est repoussée plus que jamais dans la clandestinité... et que toute écriture, tous les films qui s'intéressent à ça, sans être interdits, sont détournés du public, il y a un détournement de fond... le fond est là, la pensée est là, la capacité d'entendre cette pensée, de continuer cette réflexion sur nous-mêmes... je pense qu'elle est peut-être encore là, mais les communications ne nous appartiennent plus...

ils ont réussi à évacuer toute cette problématique, au niveau poétique, comme si ça n'avait pas d'importance... pour s'en tenir à une perception purement politique du Québec... non émotive ! non passionnelle ! une perception qui leur permet d'avoir une dialectique plus efficace au niveau de la propagande... on fait peur aux gens par tous les moyens...

il n'est pas étonnant qu'on n'arrive pas avec nos travaux à rejoindre le public... on est les créateurs, on n'a pas les moyens pour signaler aux gens notre existence...

mais tout ce que je dis c'est extrêmement tendancieux, parce qu'il est possible que les choses que j'ai faites récemment ne méritent pas que les gens les voient...

mais j'ai pas l'impression d'être le seul...

\section{LES PROJETS}

J'aime mieux pas en parler...

tu me poses la question, je t'en parle !

mais mon attitude... c'est une espèce de méfiance à l'égard de l'avenir,

je sais pas ce que j'en ferai...

et puis une tentative pour pas y penser...

je suis obligé de faire un effort pour me rappeler les titres de mes trois derniers films... j'aime mieux pas y penser... puis je te dis mes trois derniers films, c'est quatre ! j'oubliais le Gout de la farine... c'est étrange... 
très personnel au fond ce que je te dis, c'est pas bien utile pour toi probablement, parce que c'est une réflexion sur mon malaise personnel... non pas le travail que j'ai fait...

tu me poses, la question, je t'en parle !

j'ai rien de précis... j'ai une proposition de l'université Laval pour aller enseigner... j'ai pas le goût, mais enfin j'ai une proposition...

j'ai deux propositions de film en France, elles sont vagues...

des sujets qui m'intéressent sur l'Occitanie, puis la Bretagne possiblement, mais j'y vais sans enthousiasme... j'y vais sans enthousiasme...

je le fais un peu par curiosité, un peu aussi par amertume, tu sais...

je me dis faire un changement... une diversion...

remarque que je vais tourner les mêmes films... je vais tourner des films avec des gens qui sont en perte de royaume, si on peut dire...

la seule chose que je veux pas perdre, c'est la position dans laquelle je me trouve qui me permet de faire des films au Québec, parce que même si les films sont pas diffusés, je pense que c'est l'héritage que je peux laisser au Québécois, moi... c'est cette façon de le regarder dans ce moment précis qui restera dans l'histoire un moment tragique important..

tu sais...

J'aurais mieux fait de pas dire ça... parce que c'est pas utile, un propos comme ça, c'est stérilisant...

il s'est trouvé que t'es venu un soir où il y avait sans doute un peu d'amertume...

je me demande souvent si le moi a quelque importance là-dedans...

je relis ça puis je trouve ça haïssable... ! 